\title{
View on Beautiful Timbre of Urheen Performance from Technical Perspective
}

\author{
Jing Shi \\ XingTai University, Xingtai, 054001, China
}

Keywords: Urheen; Timbre; Technique

\begin{abstract}
Each musical instrument has its inherent timbre. In the actual performance of musical instruments such as urheen, the obtainment of beautiful timbre is closely related to playing techniques of urheen. Both colorful string rub technique and smooth hold shift technique can highlight the beautiful timbre of urheen performance. This paper conducts specific analysis on the obtainment of beautiful timbre in urheen performance from technical perspective based on brief analysis on the beautiful timbre of urheen and expects to enrich the theory of urheen performance.
\end{abstract}

\section{Introduction}

There is no absolute aesthetic form in the world. Beauty can only exist relatively and be identified through comparison. The form of beauty is determined by its content. There is no exception for beautiful timbre in urheen performance. It is mainly obtained through performance techniques of urheen performers. In actual urheen performance, differences in the performer' $s$ strength of pressing strings with left hand, strength of bow holding with right hand, string rub extent and bowman position can result in the change of timbre and tone quality of urheen performance. Such changes can make sound more vacant and purer or rough and dry. Therefore, it is required to master excellent performance techniques and apply timbre change manifested in urheen performance to the performance practice rationally so as to obtain rich timbre beauty and give people a unique auditory aesthetic pleasure.

\section{Analysis on timbre in urheen performance}

In the actual performance process of urheen, good timbre expression is the precondition for achieving its artistic value. Timbre of urheen has various patterns of change which can be summarized into basic timbre and emotional timbre. Basic timbres of urheen mainly refer to basic sound features of phonatory equipment in actual performance. That is to say, all sounds that can conform to the principle of sound production of urheen and that can be made through performance under the action of basic law of physiological movement of human body can be included in the list of basic timbres of urheen. For example, three timbres "Fan, San and Shi" divided in traditional music theory belong to basic timbres. "Fan" mainly refers to the sound made when the performer' s fingers press the string in empty step; "San" refers to open string in urheen performance; "Shi" refers to the sound made when the performer' s fingers press the string actually. These timbres are different, but all of them belong to basic timbres of urheen ${ }^{[1]}$. Emotional timbres in urheen performance mainly refer to the performer' s unique emotional experience for the music in the specific performance practice. The timbre of urheen is changed to a certain extent through corresponding performance technique. The specific selection of such emotional timbres is limited by the aesthetic ability and trend of performer and urheen timbre to a certain extent. Only when a performer has timbre thought in spirit during the performance can he/she show it through music. Influenced by the aesthetic trend of performers, emotional timbres have stronger personality development feature without strict right and wrong. The beauty of such timbres can be judged only through the examination on whether it can meet the demand of appreciation of appreciators. 


\section{Technical training of fingering and beautiful timbre of urheen}

Fingering technique of urheen refers to basic skills used by performers when they play a string of notes with left hand, such as finger controlling, string rub, hold shift, glide, trill, beating tone and overtone. Unique timbres of urheen are created and changed through joint control of fingering with left hand and bow use with right hand. Urheen is an instrument without fingerboard. To make the music played conform to the psychological pursuit and individual emotion of the composer, performers should select fingering reasonably. To obtain beautiful timbre in urheen performance, it is required to constantly change fingering strength and the corresponding fingering method and make timbres colorful.

\section{View on beautiful timbre of urheen from hold shift}

Due to the demand for expanding range and transforming timbre, attention should be paid to skills of hold shift in the performance of urheen. Low technical level of hold shift will definitely cause damage to the overall smoothness of music performance and magnificent effect of timbre. Hold shift and finger position are two different concepts and cannot be mixed in performance. Finger position includes traditional one and modern one, which are different. Traditional finger position of urheen includes home position, neutral position, high-order position, sub-high position and highest order. Modern finger position is a multi-finger position in essence, which can constitute the required finger position reasonably on each corresponding sound level of five-tone scale or seven-tone scale, and even constitute multiple finger positions on each smaller half sound level of equal temperament. In terms of hold shift skills in urheen performance, traditional and modern finger positions have been used rationally with differences for a long time and hold shift is completed skillfully through slide, move and bounce of left hand on urheen. The most important point in hold shift skills is to control the movement range of left hand wrist during hold shift and make it adaptive to the demand of music performance ${ }^{[2]}$. That is to say, gyration range of hold shift can be appropriately larger when the duration of music performance is long; gyration range of hold shift can be smaller when the duration is short. Moreover, in gyration pull process, left hand wrist must be relaxed and finger face pressure should be reduced appropriately so as to make the sound of urheen mellow and soft and obtain the effect of beautiful timbre.

In addition, the rhythm in hold shift is a problem to be noted in order to obtain beautiful timbre in urheen performance. Only with reasonable arrangement of time required for hold shift can the optimal timbre effect be achieved in performance. Therefore, performers should arrange the time required for hold shift between relative intervals of tones in the process of hold shift in order to effectively avoid timbre imbalance caused by inappropriate processing. Generally, it should be arranged in the tail of front tone and in front of the posterior tone adjacent to hold shift movement. When the front tone is formally converted into the posterior tone, the duration between both tones can just be controlled to the accurate value. That is to say, the posterior tone can remain a relatively complete duration on the string and the front tone should remain an appropriate duration or slide slightly on the string. Meanwhile, attention should be paid to mutual crossing of left hand movements of performers in hold shift. When the performer conducts hold shift through finger shifting or tripping skills, the left hand movement of the performer will also produce influence on the rhythm and timbre of music. Therefore, to achieve good timbre control effect, performers should control their left hand movement within the time of occurrence of open string. That is to say, besides adjusting left hand movement to the normal rhythm sensation, it is also required to control the right hand movement to the rhythm sensation so as to achieve the optimal beautiful timbre effect.

\section{View on beautiful timbre of urheen from string rub}

The main function of string rub change is retouch of timbre of urheen. The same musical note can produce multiple forms of change through different string rub methods and corresponding movement range and speed adjustment, thus making rigid timbre become full of vigor and provide urheen 
performance with strong vitality. String rub technique plays a vital role in enriching the timbre of urheen and improving the artistic expressive force and infection of urheen. Important string rub skills of urheen include pressing rub, rolling rub, buckling rub, sliding rub, and wrist rub etc. The use and combined use of these different means will produce different changes of timbre and provide the performance with rich emotional connotations. This paper will conduct specific analysis on several string rub methods below.

Pressing rub of string

Pressing rub of string is one of the component elements of basic features of urheen and contains strong national style and regional features. To be specific, finger joints of left hand bend and stretch flexibly and transfer the power to the end of fingers so as to impose certain pressure to the string, make string have appropriate tension change and produce sound wave in vibration according to certain frequency. The music effect produced by pressing rub of string generally has relatively longer sound wave and higher frequency. Therefore, emotions such as excitement, tension, sorrow or anxiety are often manifested according to its timbre features.

Wrist rub of string

In wrist rub of string, the appropriate flexion and extension of the performer' s wrist drives the palm to shake up and down rationally so as to ensure that fingers can roll up and down on the string in a relaxed way to change the length of string and produce corresponding sound wave. Wrist rub of string is a performance skill produced by Chinese urheen performance experts who have learnt and used vibrato method in violin performance techniques for reference under the influence of western culture and combined the actual performance demand of urheen. Its application can make the timbre of urheen softer and more beautiful and improve the singing effect ${ }^{[3]}$. Moreover, this performance method has a wider adaptive surface and has been widely used in modern urheen performance skills. During the practice of wrist rub of string, attention should be paid to the part of left hand fingers touching the string and the corresponding contact area which have importance influence on the change of timbre in performance. Brighter timbre can be obtained easily when the performer uses fingertip, which is a smaller part, to touch the string. The timbre can be mellow and beautiful and different timbre experience can be obtained when the performer uses finger pad, which is a larger part, to touch the string.

Sliding rub of string

Sliding rub of string is a special method of string rub. Specifically, fingers slide on the string rapidly within a certain distance so as to change the length of string and produce sound wave. Such method is not widely used in urheen performance. Generally, it must be used in the performance of some songs with intense style in the north. It has distinctive regional features and can provide the music with more outstanding timbre beauty.

No string rub

No string rub means that fingers remain static on a tone within certain time, thus producing straight timbre effect without sound wave. It constitutes strong acoustic contrast to string rub technique and has distinctive timbre comparison beauty.

\section{View on beautiful timbre of urheen performance from trill}

The flexible urheen trill technique will provide certain help for the manifestation of beautiful timbre of urheen. Trill in urheen performance generally has three main types: first, trill with stringendo prolonged sound. This trill can be played according to specific requirements in music theory and is mostly used to show beautiful natural scenery. Second, trill with sudden short sound. For the playing of this trill, it is required to first set up a preparative finger in performance practice, touch the string uniformly with fingers in original line and above the original line and then produce unique timbre effect. It is mostly used to play lively music. Third, late trill. This trill has high requirements for the flexibility of fingers. Therefore, it can make timbre more colorful. It is generally used in slow song. The performance way of trill is mainly consistent with string touch method. That is, sound is produced under the driving of finger root with clear and heavy timbre and relatively fast rhythm. 


\section{Bowing training and beautiful timbre of urheen}

The obtainment of beautiful timbre of urheen has high requirements for left hand fingering training with certain norms of science and stringency. However, the obtainment of beautiful timbre effect requires not only high fingering technique, but also bowing technical level in performance. The contact angle of bow and string, the change of bow speed vibration and the pressure of bow on string have corresponding influence on urheen timbre ${ }^{[4]}$. For example, in the performance of River of Sorrow, as one period mainly shows the sorrow and hopelessness of the young married woman in front of the surging river water after she experienced joys and sorrows in life, the performer is required to ensure that the bow produces lighter pressure on string and make the timbre give people an imaginary feeling and show somber sense. Another period shows the scene that the woman finally vented her discontent and indignation in front of the surging river water. Therefore, the performer should appropriately increase the pressure of bow on the string and make the music effect produce strong conflicts between fiction and fact through timbre change. To guarantee timbre beauty of urheen in performance, attention should be paid to the following in bowing training:

First, it is necessary to control the relaxation degree of each joint of right hand in movement. The coordination and relaxation degree of each joint of right hand is one of the factors influencing smooth bowing as well as the key to obtain smooth and relaxed timbre. The stiffness of each joint can influence power transmission in bowing.

Second, it is necessary to adjust the contact angle between bow and string. To achieve beautiful timbre, the most key point is to adjust the contact angle between bow and string well. Only with good contact angle can it drive string to produce regular vibration to the greatest extent and produce excellent consonance effect. Therefore, performers should guarantee flat, straight and steady bow and appropriate distance between bow hair and string in practice, thus allowing bow and string to produce adequate friction through full contact and produce the required vibration sound.

Third, it is necessary to control the relationship between bow speed and bow pressure. Another key bowing factor influencing urheen timbre is the relationship between bow speed and bow pressure. When pressure is higher than bow speed, the sound will be too rough; when the pressure is lower than bow speed, the sound will be too weak. Generally, the higher the bowing speed it, the smaller the pressure produced by bowing will be; otherwise, the pressure will be smaller [5]. Such speed and pressure are relative generally and can only be measured by performers through performance experience and judged and adjusted through ears. This requires trainers to gradually reach the state of "heart-hand integration" in training.

\section{Conclusion}

In conclusion, to achieve the art of beautiful timbre of urheen, it is required to conduct strict fingering training and meanwhile realize perfect combination of both hands through appropriate right hand bowing training and further transform multiple aesthetic demands of sound in music thought into actual sound effect through effective technical means. The pursuit of urheen timbre is just realized when fingers touch the string, which manifests the artistic attainment of performers and bring appreciators unique aesthetic appreciation.

\section{References}

[1] Sun Lijun. Research on Timbre of Urheen Instrument Made with MIDI Technology. Intelligence, 2014(6):300-300.

[2] Hao Jianguang. Analysis on Skilled Movement - String Rub of Urheen. Yellow River of the Song, 2013(18):110-111.

[3] Yang Si. Brief Discussion on Relationship between Tuning System Orientation and Intonation in Urheen Performance. Notonly Music, 2013(9):205-205. 
[4] Guo Xichen. Discussion on Beautiful Timbre in Urheen Performance. Charming China, 2013(31):110-110.

[5] Hu Xiaoli. Exploration on Timbre Art in Urheen Performance. Journal of Chifeng College: Chinese Philosophy and Social Science Edition, 2012(7):196-197. 